\title{
The New Methods of Improving Smooth Degree of Modeling Data Series
}

\author{
Qiuping Wang ${ }^{1}$ Jun Zhang ${ }^{2}$ \\ ${ }^{1}$ School of Business Management, Xi'an University of Technology, Xi'an 710054, P. R. China \\ ${ }^{2}$ School of Sciences, Xi'an University of Technology, Xi'an 710054, P. R. China
}

\begin{abstract}
Improving the smooth degree of modeling data series is key factor of grey model's precision. According to function transformation theory and grey system modeling theory, elementary study on $\mathrm{GM}(1,1)$ model based on function transformation is given, then the methods of linear function transformation and compound function transformation against modeling data series are put forward to improve model's precision in the paper. It has been proved that the smooth degree of modeling data series after the two new transformations can be improved. The result of practical application demonstrates effectiveness of the two new methods.
\end{abstract}

Keywords: Smooth degree, Function transformation, GM(1,1) model, Precision

\section{Introduction}

GM $(1,1)$ model [1] has been proposed by Chinese scholar professor Julong Deng in 1982. It is the most frequently used in the grey system theory. And it is also called traditional GM(1,1) model. Its precision is related to the smooth degree of modeling data series. When the smooth degree of modeling data series is not high enough, the precision of grey $\mathrm{GM}(1,1)$ model is very poor.

In order to improve the smooth degree of modeling data series, logarithm function $f=\ln x(k)$ $(x(1) \geq e)$ transformation is put forward in [2]; power transformation function $f=[x(k)]^{1 / T}(x(1) \geq$ $1, T \geq 1$ ) is put forward in [3]; logarithm-power transformation function $f=[\ln x(k)]^{1 / T}(x(1) \geq e$, $T \geq 1$ ) is put forward in [4]; transformation based on function $f=[x(k)]^{-a}(a>0)$ is put forward in [5]. And it has been proved that the several transformations can improve the smooth degree of modeling data series.

Based on the knowledge of transformation function and inverse transformation function, the methods of linear function transformation $f=p x^{(0)}(k)+q$ and compound function transformation $f=p \ln x^{(0)}(k)+q$ against modeling data series are put forward to improve the smooth degree of modeling data series. The suitable parameter $p$ and $q$ of transformation functions can be obtained by Genetic Algorithm [7] on condition that the mean absolute percentage error (MAPE) of model is minimal in [6]. Therefore, the precision of grey system model can be improved.

\section{The traditional method of improving smooth degree of modeling data series}

Definition 2.1. [8] Let $\left\{x^{(0)}(k), k=1,2, \ldots, n\right\}$ be non-negative data series, then $\rho(k)=\frac{x^{(0)}(k)}{\sum_{i=1}^{k-1} x^{(0)}(i)}$ is defined as smooth ratio of $\left\{x^{(0)}(k), k=1,2, \ldots, n\right\}$.

Definition 2.2. [8] Let $\left\{x^{(0)}(k), k=1,2, \ldots, n\right\}$ be non-negative data series, for any $\varepsilon>0$, if there exists a $k_{0}$ such that smooth ratio $\rho(k)=\frac{x^{(0)}(k)}{\sum_{i=1}^{k-1} x^{(0)}(i)}<\varepsilon$ when $k>k_{0}$, then the data series is called smooth data series.

Theorem 2.1. [8] Series $\left\{x^{(0)}(k), k=1,2, \ldots, n\right\}$ is a smooth data series iff the smooth ratio $\rho(k)$ is decrement with $k$, where $\rho(k)=\frac{x^{(0)}(k)}{\sum_{i=1}^{k-1} x^{(0)}(i)}$.

Theorem 2.2. [2] Let $\left\{x^{(0)}(k), k=1,2, \ldots, n\right\}$ be an increment data series with $k$ and $x^{(0)}(1)>e$, then 


$$
\frac{\ln x^{(0)}(k)}{\sum_{i=1}^{k-1} \ln x^{(0)}(i)} \leq \frac{x^{(0)}(k)}{\sum_{i=1}^{k-1} x^{(0)}(i)} .
$$

Theorem 2.3. [3] Let $\left\{x^{(0)}(k), k=1,2, \ldots, n\right\}$ be an increment data series with $k$ and $x^{(0)}(1) \geq 1, T \geq 1$, then $\frac{\left[x^{(0)}(k)\right]^{1 / T}}{\sum_{i=1}^{k-1}\left[x^{(0)}(i)\right]^{1 / T}} \leq \frac{x^{(0)}(k)}{\sum_{i=1}^{k-1} x^{(0)}(i)}$.

Theorem 2.4. [4] Let $\left\{x^{(0)}(k), k=1,2, \ldots, n\right\}$ be an increment data series with $k$ and $x^{(0)}(1) \geq e, T \geq 1$, then $\frac{\left[\ln x^{(0)}(k)\right]^{1 / T}}{\sum_{i=1}^{k-1}\left[\ln x^{(0)}(i)\right]^{1 / T}} \leq \frac{\ln x^{(0)}(k)}{\sum_{i=1}^{k-1} \ln x^{(0)}(i)}$.

Theorem 2.5. [5] Let $\left\{x^{(0)}(k), k=1,2, \ldots, n\right\}$ be an increment data series with $k$ and $x^{(0)}(1) \geq e, T \geq 1$, then for $a>0$, the following inequality is true.

$$
\begin{aligned}
& \frac{\left[x^{(0)}(k)\right]^{-a}}{\sum_{i=1}^{k-1}\left[x^{(0)}(i)\right]^{-a}} \leq \frac{\left[\ln x^{(0)}(k)\right]^{1 / T}}{\sum_{i=1}^{k-1}\left[\ln x^{(0)}(i)\right]^{1 / T}} \\
& \leq \frac{\ln x^{(0)}(k)}{\sum_{i=1}^{k-1} \ln x^{(0)}(i)} \leq \frac{x^{(0)}(k)}{\sum_{i=1}^{k-1} x^{(0)}(i)}
\end{aligned}
$$

\section{The method of improving smooth degree of modeling data series based on linear function transformation}

Theorem 3.1. Let $\left\{x^{(0)}(k), k=1,2, \ldots, n\right\}$ be an increment data series with $k$ and $x^{(0)}(1)>0$, then $\left\{p x^{(0)}(k)+q\right\}$ is smooth data series, where $p<0$, $q>0, p x^{(0)}(k)+q>0, k=1,2, \ldots, n$.

Proof. Because series $\left\{x^{(0)}(k), k=1,2, \ldots, n\right\}$ is an increment data series with $k$ and $x^{(0)}(1)>0$, $\left\{p x^{(0)}(k)+q\right\}$ is a non-negative decrement data series, where $p<0, q>0, p x^{(0)}(k)+q>0, k=1$, $2, \ldots, n$.
We have $0<p x^{(0)}(k+1)+q<p x^{(0)}(k)+q$ and $0<\sum_{i=1}^{k-1}\left[p x^{(0)}(i)+q\right]<\sum_{i=1}^{k}\left[p x^{(0)}(i)+q\right]$.

Thus $\left[p x^{(0)}(k+1)+q\right] \sum_{i=1}^{k-1}\left[p x^{(0)}(i)+q\right]$

$$
<\left[p x^{(0)}(k)+q\right] \sum_{i=1}^{k}\left[p x^{(0)}(i)+q\right] .
$$

Namely

$$
\frac{p x^{(0)}(k+1)+q}{\sum_{i=1}^{k}\left[p x^{(0)}(i)+q\right]}<\frac{p x^{(0)}(k)+q}{\sum_{i=1}^{k-1}\left[p x^{(0)}(i)+q\right]} .
$$

Therefore

$$
\left\{p x^{(0)}(k)+q\right\}\left(p<0, q>0, p x^{(0)}(k)+q>0\right)
$$

is smooth data series according to Theorem 2.1.

Theorem 3.2. Let series $\left\{x^{(0)}(k), k=1,2, \ldots, n\right\}$ be an increment data series with $k$ and $x^{(0)}(1)>0, p<$ $0, q>0, p x^{(0)}(k)+q>0, k=1,2, \ldots, n$.

Then $\frac{p x^{(0)}(k)+q}{\sum_{i=1}^{k-1}\left[p x^{(0)}(i)+q\right]} \leq \frac{x^{(0)}(k)}{\sum_{i=1}^{k-1} x^{(0)}(i)}$.

Proof. Because series $\left\{x^{(0)}(k), k=1,2, \ldots, n\right\}$ is a non-negative increment data series with $k$, we get

$$
0<x^{(0)}(i) \leq x^{(0)}(k)(i=1,2, \ldots, k-1) .
$$

And $x^{(0)}(1)>0,\left\{p x^{(0)}(k)+q\right\}(p<0, q>0$, $\left.p x^{(0)}(k)+q>0, k=1,2, \ldots, n\right)$ is a non-negative decrement series with $k$, thus

$0<p x^{(0)}(k)+q \leq p x^{(0)}(i)+q(i=1,2, \ldots, k-1)$. We have

$$
\begin{aligned}
& x^{(0)}(i)\left[p x^{(0)}(k)+q\right] \leq x^{(0)}(k)\left[p x^{(0)}(i)+q\right] \\
& (i=1,2, \ldots, k-1) .
\end{aligned}
$$

Thus

$\sum_{i=1}^{k-1} x^{(0)}(i)\left[p x^{(0)}(k)+q\right] \leq \sum_{i=1}^{k-1} x^{(0)}(k)\left[p x^{(0)}(i)+q\right]$.

Namely

$\left[p x^{(0)}(k)+q\right] \sum_{i=1}^{k-1} x^{(0)}(i) \leq x^{(0)}(k) \sum_{i=1}^{k-1}\left[p x^{(0)}(i)+q\right]$.

Therefore

$$
\frac{p x^{(0)}(k)+q}{\sum_{i=1}^{k-1}\left[p x^{(0)}(i)+q\right]} \leq \frac{x^{(0)}(k)}{\sum_{i=1}^{k-1} x^{(0)}(i)} .
$$


In the end, Theorem 3.2 shows the smooth degree of data series $\left\{p x^{(0)}(k)+q\right\}$ is better than that of original data series $\left\{x^{(0)}(k)\right\}$, where $p<0, q>0$, $p x^{(0)}(k)+q>0, k=1,2, \ldots, n$.

\section{Modeling method based on linear function transformation}

\subsection{Main thought of modeling method based on linear function transformation

$$
f=p x^{(0)}(k)+q
$$

Series $\left\{x^{(0)}(k), k=1,2, \ldots, n\right\}$ is original data series. Let $y^{(0)}(k)=p x^{(0)}(k)+q, k=1,2, \ldots, n$, then the parameter $p$ and $q$ of transformation function can be obtained by Genetic Algorithm under the criterion of minimization $\frac{1}{n} \sum_{k=1}^{n}\left|\frac{\hat{x}^{(0)}(k)-x^{(0)}(k)}{x^{(0)}(k)}\right|$ after inverse transformation $\hat{x}^{(0)}(k)=\frac{\hat{y}^{(0)}(k)-q}{p}$.

\subsection{Main steps of modeling method based on linear function transformation $f=p x^{(0)}(k)+q$}

Step 1: The original data series $\left\{x^{(0)}(k)\right\}$ is transformed into new data series $\left\{p x^{(0)}(k)+q\right\}$, which is denoted by $\left\{y^{(0)}(k)\right\}(k=1,2, \ldots, n)$.

Step 2: New series $\left\{y^{(0)}(k), k=1,2, \ldots, n\right\}$ is used to establish traditional $\operatorname{GM}(1,1)$ model [1], then forecasting formula:

$$
\begin{aligned}
& \hat{y}^{(0)}(1)=y^{(0)}(1)=p x^{(0)}(1)+q \\
& \hat{y}^{(0)}(k)=\left(1-e^{\hat{a}}\right)\left(y^{(0)}(1)-\hat{b} / \hat{a}\right) e^{-\hat{a}(k-1)} \\
& k=2,3, \ldots
\end{aligned}
$$

is obtained by calculation, where $\hat{a}$ is estimate value of developing coefficient and $\hat{b}$ is estimate value of the grey input.

Step 3: According to inverse transformation function $\hat{x}^{(0)}(k)=\frac{\hat{y}^{(0)}(k)-q}{p}$, forecasting formula:

$$
\begin{aligned}
& \hat{y}^{(0)}(1)=y^{(0)}(1)=p x^{(0)}(1)+q \\
& \hat{y}^{(0)}(k)=\left(1-e^{\hat{a}}\right)\left(y^{(0)}(1)-\hat{b} / \hat{a}\right) e^{-\hat{a}(k-1)} \\
& k=2,3, \ldots
\end{aligned}
$$

can be turned into

$$
\hat{x}^{(0)}(1)=x^{(0)}(1)
$$

$$
\hat{x}^{(0)}(k)=\frac{\left(1-e^{\hat{a}}\right)\left(p x^{(0)}(1)+q-\frac{\hat{b}}{\hat{a}}\right) e^{-\hat{a}(k-1)}-q}{p}
$$

$k=2,3, \ldots$

\subsection{Application of modeling method based on linear function transformation$$
f=p x^{(0)}(k)+q
$$

Per capita annual net income of rural households is an important index of national agriculture economic development level. Establishing grey forecasting model of Per capita annual net income of rural households and forecasting its future trend will have great significance. First, linear function transformational GM(1,1) model of Per capita annual net income of rural households from 1996 to 2003 is established. Then, Per capita annual net income of rural households from 2004 to 2005 is forecasted. (Data form “China Statistical Yearbook-2006” [9])

(1) The traditional GM(1,1) model of these data is as follows:

$$
\begin{aligned}
& \hat{x}^{(0)}(1)=x^{(0)}(1)=1926.1 \\
& \hat{x}^{(0)}(k)=1986.0 e^{0.0372(k-1)} \\
& k=2,3, \ldots
\end{aligned}
$$

where

$$
\hat{a}=-0.0372, \hat{b}=1951.5 \text {. }
$$

(2) The model based on linear function transformation $f=p x^{(0)}(k)+q$ is as follows:

$$
\begin{aligned}
& \hat{x}^{(0)}(1)=x^{(0)}(1)=1926.1 \\
& \hat{x}^{(0)}(k)=47.9429 e^{0.3635(k-1)}+2058.3 \\
& k=2,3, \ldots
\end{aligned}
$$

where

$$
\begin{aligned}
& \hat{a}=-0.3635, \hat{b}=-18.8084, \\
& p=-0.1787, q=367.8248 .
\end{aligned}
$$

Table 1 shows that the mean absolute percentage error and forecasting relative error of the model based on linear function transformation $f=p x^{(0)}(k)+q$ are much less than that of traditional $\operatorname{GM}(1,1)$ model. In a word, it is obvious that the modeling method 
based on linear function transformation has improved

model. the fitted precision and forecasting precision of grey

\begin{tabular}{|c|c|c|c|c|c|c|}
\hline \multirow{2}{*}{ No. } & \multirow{2}{*}{ Year } & \multirow{2}{*}{$\begin{array}{l}\text { Per capita annual } \\
\text { net income of } \\
\text { rural households }\end{array}$} & \multicolumn{2}{|c|}{$\begin{array}{c}\text { Traditional GM(1,1) } \\
\text { model }\end{array}$} & \multicolumn{2}{|c|}{$\begin{array}{l}\text { Function } p x^{(0)}(k)+q \\
\text { transformational model }\end{array}$} \\
\hline & & & $\begin{array}{l}\text { Model } \\
\text { values }\end{array}$ & $\begin{array}{c}\text { Relative } \\
\text { error }\end{array}$ & $\begin{array}{l}\text { Model } \\
\text { values }\end{array}$ & $\begin{array}{c}\text { Relative } \\
\text { error }\end{array}$ \\
\hline 1 & 1996 & 1926.1 & 1926.1 & $0 \%$ & 1926.1 & $0 \%$ \\
\hline 2 & 1997 & 2090.1 & 2061.3 & $1.38 \%$ & 2127.3 & $-1.78 \%$ \\
\hline 3 & 1998 & 2162.0 & 2139.4 & $1.05 \%$ & 2157.5 & $0.21 \%$ \\
\hline 4 & 1999 & 2210.3 & 2220.5 & $-0.46 \%$ & 2201.0 & $0.42 \%$ \\
\hline 5 & 2000 & 2253.4 & 2304.6 & $-2.27 \%$ & 2263.5 & $-0.45 \%$ \\
\hline 6 & 2001 & 2366.4 & 2392.0 & $-1.08 \%$ & 2353.5 & $0.55 \%$ \\
\hline 7 & 2002 & 2475.6 & 2482.6 & $-0.28 \%$ & 2482.9 & $-0.29 \%$ \\
\hline 8 & 2003 & 2622.2 & 2576.7 & $1.74 \%$ & 2669.0 & $-1.78 \%$ \\
\hline \multicolumn{3}{|c|}{ MAPE } & \multicolumn{2}{|c|}{$1.18 \%$} & \multicolumn{2}{|c|}{$0.78 \%$} \\
\hline 9 & $2004^{*}$ & 2936.4 & 2674.4 & $8.92 \%$ & 2936.7 & $-0.01 \%$ \\
\hline 10 & $2005^{*}$ & 3254.9 & 2775.8 & $14.72 \%$ & 3321.7 & $-2.05 \%$ \\
\hline
\end{tabular}

(*Forecasting value)

Table 1: Comparison of two modeling methods (Unit: Yuan).

\section{The method of improving smooth degree of data series based on compound transformation}

Theorem 5.1. Let $\left\{x^{(0)}(k), k=1,2, \ldots, n\right\}$ be an increment data series with $k$ and $x^{(0)}(1)>0$, then $\left\{p \ln x^{(0)}(k)+q\right\}$ is a smooth data series, where $p<0, q>0, p \ln x^{(0)}(k)+q>0, k=1,2, \ldots, n$. Proof. Since $\left\{x^{(0)}(k)\right\}$ is an increment series with $k$ and $x^{(0)}(1)>e,\left\{p \ln x^{(0)}(k)+q\right\}$ is a non-negative decrement series, where $p \ln x^{(0)}(k)+q>0, p<0$, $q>0, k=1,2, \ldots, n$.

We have and

$$
0<p \ln x^{(0)}(k+1)+q<p \ln x^{(0)}(k)+q
$$$$
0<\sum_{i=1}^{k-1}\left[p \ln x^{(0)}(i)+q\right]<\sum_{i=1}^{k}\left[p \ln x^{(0)}(i)+q\right] .
$$

Thus

$$
\begin{aligned}
& {\left[p \ln x^{(0)}(k+1)+q\right] \sum_{i=1}^{k-1}\left[p \ln x^{(0)}(i)+q\right]} \\
& <\left[p \ln x^{(0)}(k)+q\right] \sum_{i=1}^{k}\left[p \ln x^{(0)}(i)+q\right] .
\end{aligned}
$$

Namely

$$
\frac{p \ln x^{(0)}(k+1)+q}{\sum_{i=1}^{k}\left[p \ln x^{(0)}(i)+q\right]}<\frac{p \ln x^{(0)}(k)+q}{\sum_{i=1}^{k-1}\left[p \ln x^{(0)}(i)+q\right]} .
$$

Therefore $\left\{p \ln x^{(0)}(k)+q\right\}$ is a smooth data series according to theorem 2.1, where $p \ln x^{(0)}(k)+q>$ $0, p<0, q>0, k=1,2, \ldots, n$.

Theorem 5.2. Let $\left\{x^{(0)}(k), k=1,2, \ldots, n\right\}$ be an increment data series with $k$ and $x^{(0)}(1)>e, p<0$, $q>0, p \ln x^{(0)}(k)+q>0, k=1,2, \ldots, n$, then $\frac{p \ln x^{(0)}(k)+q}{\sum_{i=1}^{k-1}\left[p \ln x^{(0)}(i)+q\right]} \leq \frac{\ln x^{(0)}(k)}{\sum_{i=1}^{k-1} \ln x^{(0)}(i)} \leq \frac{x^{(0)}(k)}{\sum_{i=1}^{k-1} x^{(0)}(i)}$ Proof. According to Theorem 2.2, the second inequality in theorem 5.2 is true. We only need to prove the first inequality in theorem 5.2.

Because data series $\left\{x^{(0)}(k), k=1,2, \ldots, n\right\}$ is a non-negative increment data series with $k$, we get

$$
\begin{aligned}
& 0<x^{(0)}(i) \leq x^{(0)}(k)(i=1,2, \ldots, k-1), \\
& 0<\ln x^{(0)}(i) \leq \ln x^{(0)}(k)(i=1,2, \ldots, k-1) .
\end{aligned}
$$

And $\left\{p \ln x^{(0)}(k)+q\right\}$ is a non-negative decrement data series with $k$, where $p \ln x^{(0)}(k)+q>0, p<$ $0, q>0, k=1,2, \ldots, n$.

Thus 


$$
\begin{aligned}
& 0<p \ln x^{(0)}(k)+q \leq p \ln x^{(0)}(i)+q \\
& (i=1,2, \ldots, k-1) .
\end{aligned}
$$

We have

$$
\begin{aligned}
& \ln x^{(0)}(i)\left[p \ln x^{(0)}(k)+q\right] \\
& \leq \ln x^{(0)}(k)\left[p \ln x^{(0)}(i)+q\right], \\
& (i=1,2, \ldots, k-1) .
\end{aligned}
$$

Thus

$$
\begin{aligned}
& \sum_{i=1}^{k-1} \ln x^{(0)}(i)\left[p \ln x^{(0)}(k)+q\right] \\
& \leq \sum_{i=1}^{k-1} \ln x^{(0)}(k)\left[p \ln x^{(0)}(i)+q\right] .
\end{aligned}
$$

Namely

$$
\begin{aligned}
& {\left[p \ln x^{(0)}(k)+q\right] \sum_{i=1}^{k-1} \ln x^{(0)}(i)} \\
& \leq \ln x^{(0)}(k) \sum_{i=1}^{k-1}\left[p \ln x^{(0)}(i)+q\right] .
\end{aligned}
$$

Therefore

$$
\frac{p \ln x^{(0)}(k)+q}{\sum_{i=1}^{k-1}\left[p \ln x^{(0)}(i)+q\right]} \leq \frac{\ln x^{(0)}(k)}{\sum_{i=1}^{k-1} \ln x^{(0)}(i)} .
$$

Theorem 5.2 shows the smooth degree of series $\left\{p \ln x^{(0)}(k)+q\right\}\left(p<0, q<0, p \ln x^{(0)}(k)+q>0\right)$ is better than that of data series $\left\{\ln x^{(0)}(k)\right\}$ and $\left\{x^{(0)}(k)\right\}(k=1,2, \ldots n)$.

\section{Modeling method based on compound function transformation}

\subsection{Main thought of modeling method based on compound function transformation}

Series $\left\{x^{(0)}(k), k=1,2, \ldots, n\right\}$ is original data series, let $y^{(0)}(k)=p \ln x^{(0)}(k)+q, k=1,2, \ldots, n$, the parameter $p$ and $q$ of transformation function can be obtained by Genetic Algorithm under the criterion of minimization $\frac{1}{n} \sum_{k=1}^{n}\left|\frac{\hat{x}^{(0)}(k)-x^{(0)}(k)}{x^{(0)}(k)}\right|$ after inverse transformation $\hat{x}^{(0)}(k)=e^{\left[\hat{y}^{(0)}(k)-q\right] / p}$.

\subsection{Main steps of modeling method based on compound function transformation}

Step 1: The original data series $\left\{x^{(0)}(k)\right\}$ is transformed into new data series $\left\{p \ln x^{(0)}(k)+q\right\}$ which is denoted by $\left\{y^{(0)}(k)\right\}(k=1,2, \ldots, n)$.

Step 2: New data series $\left\{y^{(0)}(k)\right\}$ is used to establish traditional GM(1,1) model [1], forecasting formula:

$$
\begin{aligned}
& \hat{y}^{(0)}(k)=y^{(0)}(1)=p \ln x^{(0)}(1)+q \\
& \hat{y}^{(0)}(k)=\left(1-e^{\hat{a}}\right)\left(y^{(0)}(1)-\hat{b} / \hat{a}\right) e^{-\hat{a}(k-1)} \\
& k=2,3, \ldots
\end{aligned}
$$

is obtained by calculation.

Step 3: According to inverse transformation function $\hat{x}^{(0)}(k)=e^{\left[\hat{y}^{(0)}(k)-q\right] / p}$, forecasting formula:

$$
\begin{aligned}
& \hat{y}^{(0)}(k)=y^{(0)}(1)=p \ln x^{(0)}(1)+q \\
& \hat{y}^{(0)}(k)=\left(1-e^{\hat{a}}\right)\left(y^{(0)}(1)-\hat{b} / \hat{a}\right) e^{-\hat{a}(k-1)} \\
& k=2,3, \ldots
\end{aligned}
$$

can be turned into

$$
\begin{aligned}
& \hat{x}^{(0)}(k)=x^{(0)}(1) \\
& \hat{x}^{(0)}(k)=e^{\left[\left(1-e^{\hat{a}}\right)\left(p \ln x^{(0)}(1)+q-\frac{\hat{b}}{\hat{a}}\right) e^{-\hat{a}(k-1)}-q\right] / p} \\
& k=2,3, \ldots .
\end{aligned}
$$

\subsection{Application of modeling method based on compound function transformation}

Now compound function transformational $\operatorname{GM}(1,1)$ model of China annual per capita electricity consumption from 1996 to 2003 is established, and China annual per capita electricity consumption from 2004 to 2005 is forecasted. (Data form "China Statistical Yearbook-2006” [9])

(1) The traditional GM $(1,1)$ model of these data is as follows:

$$
\begin{aligned}
& \hat{x}^{(0)}(1)=x^{(0)}(1)=93.1 \\
& \hat{x}^{(0)}(k)=90.5396 e^{0.0923(k-1)} \\
& k=2,3, \ldots
\end{aligned}
$$

where

$$
\hat{a}=-0.0923, \hat{b}=86.1891
$$

(2) The model based on logarithm function transformation $f=\ln x^{(0)}(k)$ is as follows: 


$$
\begin{aligned}
& \hat{x}^{(0)}(1)=x^{(0)}(1)=93.1 \\
& \hat{x}^{(0)}(k)=e^{4.5189 e^{0.0188(k-1)}} \\
& k=2,3, \ldots
\end{aligned}
$$

where

$$
\hat{a}=-0.0188, \hat{b}=4.4763
$$

(3) The model based on compound function transformation $f=p \ln x^{(0)}(k)+q$ is as follows:

\begin{tabular}{|c|c|c|c|c|c|c|c|c|}
\hline \multirow[t]{2}{*}{ No. } & \multirow[t]{2}{*}{ Year } & \multirow{2}{*}{$\begin{array}{c}\text { China } \\
\text { annual per } \\
\text { capita } \\
\text { electricity } \\
\text { consumption }\end{array}$} & \multicolumn{2}{|c|}{$\begin{array}{c}\text { Traditional } \\
\text { GM(1,1) model }\end{array}$} & \multicolumn{2}{|c|}{$\begin{array}{c}\text { Function } \\
\ln x^{(0)}(k) \\
\text { transformational } \\
\text { model }\end{array}$} & \multicolumn{2}{|c|}{$\begin{array}{c}\text { Function } \\
p \ln x^{(0)}(k)+q \\
\text { transformational } \\
\text { model }\end{array}$} \\
\hline & & & $\begin{array}{l}\text { Model } \\
\text { values }\end{array}$ & $\begin{array}{l}\text { Relative } \\
\text { error }\end{array}$ & $\begin{array}{l}\text { Model } \\
\text { values }\end{array}$ & $\begin{array}{l}\text { Relative } \\
\text { error }\end{array}$ & $\begin{array}{l}\text { Model } \\
\text { values }\end{array}$ & $\begin{array}{c}\text { Relative } \\
\text { error }\end{array}$ \\
\hline 1 & 1996 & 93.1 & 93.1 & $0 \%$ & 93.1 & $0 \%$ & 93.1 & $0 \%$ \\
\hline 2 & 1997 & 101.8 & 99.29 & $2.47 \%$ & 99.95 & $1.82 \%$ & 100.15 & $1.62 \%$ \\
\hline 3 & 1998 & 106.6 & 108.90 & $-2.16 \%$ & 109.08 & $-2.33 \%$ & 109.13 & $-2.37 \%$ \\
\hline 4 & 1999 & 118.2 & 119.42 & $-1.03 \%$ & 119.23 & $-0.87 \%$ & 119.19 & $-0.84 \%$ \\
\hline 5 & 2000 & 132.4 & 130.97 & $1.08 \%$ & 130.56 & $1.39 \%$ & 130.48 & $1.45 \%$ \\
\hline 6 & 2001 & 144.6 & 143.64 & $0.66 \%$ & 143.21 & $0.96 \%$ & 143.17 & 0.99\% \\
\hline 7 & 2002 & 156.3 & 157.53 & $-0.79 \%$ & 157.35 & $-0.67 \%$ & 157.48 & $-0.75 \%$ \\
\hline 8 & 2003 & 173.7 & 172.76 & $0.54 \%$ & 173.21 & $0.28 \%$ & 173.65 & $0.03 \%$ \\
\hline \multicolumn{3}{|c|}{ MAPE } & \multicolumn{2}{|c|}{$1.25 \%$} & \multicolumn{2}{|c|}{$1.19 \%$} & \multicolumn{2}{|c|}{$1.15 \%$} \\
\hline 9 & $2004^{*}$ & 190.2 & 189.46 & $-0.39 \%$ & 191.01 & $0.43 \%$ & 191.98 & $0.94 \%$ \\
\hline 10 & $2005^{*}$ & 216.7 & 207.78 & $-4.12 \%$ & 211.03 & $-2.62 \%$ & 212.79 & $-1.80 \%$ \\
\hline
\end{tabular}

$$
\begin{aligned}
& \hat{x}^{(0)}(1)=x^{(0)}(1)=93.1 \\
& \hat{x}^{(0)}(k)=e^{3.2039 e^{0.0258(k-1)}+1.3190} \\
& k=2,3, \ldots
\end{aligned}
$$

where

$$
\begin{aligned}
& \hat{a}=-0.0258, \hat{b}=-0.6891 \\
& p=-0.2179, q=0.2874 .
\end{aligned}
$$

(*Forecasting value)

Table 2: Comparison of three modeling methods (Unit: kwh).

Table 2 shows that the mean absolute percentage error (MAPE) and forecasting relative error of the model based on compound function transformation $f=p \ln x^{(0)}(k)+q$ are less than that of traditional $\operatorname{GM}(1,1)$ model. In a word, it is obvious that modeling method based on compound function transformation $f=p \ln x^{(0)}(k)+q$ has improved the fitted precision and forecasting precision of grey model.

\section{Conclusions}

In the paper, Based on function transformation theory, two new function transformation methods of improving smooth degree of modeling data series are proposed. It has been proved that smooth degree of modeling data series after the two transformations is better than that of the original data series. The result of two examples in the paper shows that the precision of model after the two transformations is better than that of traditional $\mathrm{GM}(1,1)$ model.

Additionally, the methods of improving smooth degree of modeling data series proposed in the paper are only sufficient and not necessary condition of improving grey model's precision. There are other transformational methods of improving smooth degree of modeling data series to improve grey model's precision. And the two new modeling methods based on function transformation are only suitable for shortterm prediction, and long-term forecasting model need to research in the future.

\section{Acknowledgement}

The authors wish to thank the proceedings editors and the two anonymous reviewers for their helpful comments and suggestions on the presentation of this manuscript. 


\section{References}

[1] J.L. Deng, Grey Prediction and Grey Decision,Wuhan: Huazhong University of Science and Technology Press, 2002.

[2] T.J. Chen, An Expansion of Grey Prediction Model. System Engineering, 8(4): 50-52, 1990.

[3] Q. Li, The Further Expansion of Grey Prediction Model. Systems Engineering-Theory \& Practice, 13(1):64-66, 1993.

[4] B. He, Q. Meng, Research on Methods of Expending Grey Prediction Model. Systems Engineering-Theory \& Practice, 22(9): 138-141, 2002.

[5] W.Z. Dai, J.F. Li, GM(1,1) Model Based on Power Function $X^{-a}$ Transformation and It's Application in Chinese Country Per-capita
Housing Areas Modeling. Systems EngineeringTheory \& Practice, 24(11): 63-67, 2004.

[6] T. Min, X.D. Zhou, M.Q. Feng, Inverse Problem of Multiple Parameters Identification for Water Quality Model Using Evolutionary Algorithm. Journal of Hydraulic Engineering, 10: 119-123, 2003.

[7] X.Q. Wang, L.M. Cao, Genetic Algorithms, Xi'an: Xi'an Jiaotong University Press, 2002.

[8] S.F. Liu, T.B. Guo, Y.G. Dang, Theory and Application of Grey System, Beijing: Science Press, 1999.

[9] http://www.stats.gov.cn/tjjj/ndsj/2006/indexch.ht $\mathrm{m}$.

[10] Q.M. Chen, W.Z. Dai, The General Method of Improving Smooth Degree of Data Series. International Conference on Intelligent Computing. ICIC(1), PP.505-513, 2005. 